\title{
COMPOSICIÓN QUÍMICA, ACTIVIDAD ANTIBACTERIANA DEL ACEITE ESENCIAL DE Citrus sinensis L. (NARANJA DULCE) Y FORMULACIÓN DE UNA FORMA FARMACÉUTICA
}

\author{
Chemical composition, antibacterial activity of essential oil Citrus sinensis 1. (Sweet orange) \\ and formulation of a pharmaceutical form
}

José R. Juárez, Américo J. Castro, José F. Jaúregui, Jesús V. Lizano, Mario Carhuapoma, Fritz F. Choquesillo, Luis M. Félix, Pedro A. Cotillo, Julio P. López, Marilú R. Jaramillo, Augusta I. Córdova, Julio R. Ruíz, Norma J. Ramos. Facultad de Farmacia y Bioquímica, Universidad Nacional Mayor de San Marcos.

\section{RESUMEN}

El presente estudio tuvo como objetivos, caracterizar los componentes químicos del aceite esencial de Citrus sinensis $\mathrm{L}$. (Naranja dulce), determinar su actividad antibacteriana y formular formas farmacéuticas, principalmente de uso local. El aceite esencial se obtuvo del pericarpio , de la especie seleccionada, utilizando un sistema de hidrodestilación con arrastre de vapor de agua; así mismo, se realizaron los ensayos de miscibilidad y determinación de constantes físicas, como: gravedad específica, índice de refracción y rotación óptica. La composición química se determinó por Cromatografía de Gases/Espectrometría de Masa (CG/ EM), destacándose en el cromatograma señales espectrales de identificación en la elucidación de: Limoneno, ß̧-linalol, decanal y 2(10)-pineno(1S,5S)-(-)-. La determinación de la actividad antibacteriana in vitro se realizó por el método de difusión en agar y en la formación de halos de inhibición contra cepas de microorganismos Gram (+), como: Staphylococcus aureus ATCC 25933 y Staphylococcus epidermidis, demostrando actividad antibacteriana significativa a concentraciones de 100 y 50\%, respectivamente; no así con los microorganismos Gram (-): Bacillus subtilis, Escherichia coli y Pseudomonas aeruginosa ATCC 27853. Estos resultados nos dieron la orientación para diseñar formas farmacéuticas de uso local, formulándose: un gel con base de carbomer, una loción con base de etanol y alcohol isopropílico, así como un colutorio. Los resultados de los estudios de estabilidad de las formulaciones, así como de tolerancia e irritabilidad con el tejido humano de los productos nos muestran resultados favorables para su utilización en enfermedades de la piel, como el acné, y en el caso del colutorio como un buen antiséptico bucal. El estudio ha permitido concluir que el aceite esencial de Citrus sinensis L. posee actividad antibacteriana contra cepas de Staphylococcus aureus ATCC 25933 y Staphylococcus epidermidis, además de permitir la elaboración de formas farmacéuticas de uso local.

Palabras clave: Aceite esencial, Citrus sinensis L., formas farmacéuticas.

\section{SUMMARY}

This study aimed to characterize the chemical components of essential oil of Citrus sinensis L., determine its antibacterial activity and make dosageforms, mainly for local use. The essential oil was obtained from the dried leaves of the selected species, using a hydrodistillation system with water steam sweep; tests were conducted miscibility and determination of physical constants, such as specific gravity, refractive index and optical rotation. The chemical composition was determined by Gas Chromatography/Mass Spectrometry (CG/EM), standing out in the sugiero: chromatogram spectral signals in the elucidating: Limonene, beta-linalool, decanal and 2 (10)-pinene $(1 \mathrm{~S}, 5 \mathrm{~S})-(-)-$. The determination of antibacterial activity in vitro was tested by agar diffusion method and the formation of inhibition halos against strains of Gram (+), as Staphylococcus aureus ATCC 25,933 and Staphylococcus epidermidis, demonstrating significant antibacterial activity at concentrations 100 and $50 \%$ respectively, but not with Gram (-): Bacillus subtilis, Escherichia coli and Pseudomonas aeruginosa ATCC 27853. These results gave us the guidance to design dosage forms for local use, formulated: a carbomer based gel, a lotion based in ethanol and isopropyl alcohol and mouthwash. The results of the stability studies of the formulations, as well as tolerance and irritability with human tissue of the products show favorable results for use in skin diseases like acne, and in the case of mouthwash as a good oral antiseptic. The study has revealed that the essential oil of Citrus sinensis L. has antibacterial activity against strains of Staphylococcus aureus ATCC 25,933 and Staphylococcus epidermidis, also allow the preparation of pharmaceutical forms for local use.

Key words: essential oil, Citrus sinensis L., pharmaceutical forms.

\section{INTRODUCCIÓN}

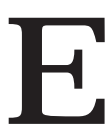

xisten sustancias naturales que pueden ser utilizadas en enfermedades de la piel y afecciones de la cavidad bucal. Dentro de éstas hallamos a los aceites esenciales que, por las propiedades que se les atribuyen, ejercen acción antioxidante, antiviral, antimicótica, repelente, antitumoral y antibacteriana. Estas sustancias han entrado a competir con sucedáneos sintéticos que pueden presentar 
efectos nocivos para la salud. Los aceites esenciales son sustancias orgánicas constituidas por terpenos, sesquiterpenos y compuestos aromáticos que se localizan en determinados órganos de la planta como flores, hojas y frutos; y se les obtiene por destilación por arrastre con vapor de agua dependiendo del método y la condición del vegetal, comportándose como líquidos miscibles en solventes orgánicos e inmiscibles en agua. Los objetivos del presente estudio son: caracterizar la composición química del aceite esencial del pericarpio, determinar su actividad antibacteriana, diseñar y formular formas farmacéuticas de aplicación local, y realizar pruebas de estabilidad.

\section{MATERIALES Y MÉTODOS}

El diseño del estudio es de tipo analítico, experimental, transversal y prospectivo, habiéndose realizado en los laboratorios del Instituto de Ciencias Farmacéuticas y Recursos Naturales "Juan de Dios Guevara" y del Instituto de Química Biológica, Microbiología y Biotecnología "Marco Antonio Garrido Malo" de la Facultad de Farmacia y Bioquímica de la Universidad Nacional Mayor de San Marcos.

\section{Material biológico}

La muestra se obtuvo en el Mercado Central de Lima.

\section{Extracción del aceite esencial}

Obtenido el pericarpio, éste se sometió a un proceso de hidrodestilación por arrastre con vapor de agua, obteniéndose un rendimiento de 0,4 por ciento $\mathrm{p} / \mathrm{v}$. Posteriormente se realizó el análisis organoléptico, ensayo de miscibilidad y propiedades fisicoquímicas.

\section{Análisis por Cromatografía de Gas/ Espectrómetro de Masa (CG/EM)}

La determinación de los componentes químicos del aceite esencial por CG/EM, se realizó en las siguientes condiciones: Columna silicagel fundida 30 metros de largo, temperatura inicial $40^{\circ} \mathrm{C}, 10^{\circ} \mathrm{C} / \mathrm{min}$, temperatura final $110^{\circ} \mathrm{C}(1 \mathrm{~min})$, inyección $240^{\circ} \mathrm{C}$ y volumen de inyección $5 \mu \mathrm{L}$. Mediante este sistema se detectaron y elucidaron los componentes químicos del aceite esencial, los que se identificaron por comparación con los estándares de espectros de masas de las respectivas bibliotecas.

\section{Determinación de la actividad antibacteriana in vitro}

Se realizó por el método de difusión en agar, midiéndose los halos de inhibición frente a cepas de microoganismos Gram positivos ${ }^{(1,2)}$ : Staphylococcus au- reus ATCC 25933, Staphylococcus epidermidis (cepa clínica), Bacillus subtilis (cepa clínica), Escherichia coli (cepa clínica) y Pseudomonas aeruginosa ATCC 27853.

\section{Formulación de formas farmacéuticas}

Considerando los resultados de los ensayos microbiológicos, mediante los cuales se demostró la actividad del aceite esencial sobre los microorganismos seleccionados, se procedió al diseño, formulación y elaboración de los siguientes tipos de productos farmacéuticos: gel hidroalcohólico, loción para la piel y colutorio, a los que se les incorporó concentraciones variadas del aceite esencial.

\section{Formulación yelaboración de formas farmacéuticas}

Se ensayaron diferentes tipos de formulaciones a base de diferentes gelificantes, como carboximetilcelulosa sódica, carbómeros y mezclas de ambos con otros agentes, como las gomas; así como para la loción y colutorio, quedando definidas las siguientes formulaciones:

\begin{tabular}{|c|c|}
\hline \multicolumn{2}{|l|}{ Gel } \\
\hline Carbómero 940 & $1 \%$ \\
\hline Trietanolamina & $0.30 \%$ \\
\hline Alcohol isopropílico & $20 \%$ \\
\hline Etanol & $40 \%$ \\
\hline Aceite esencial de naranja & $1 \%$ \\
\hline Agua c.s.p. & $100 \mathrm{~mL}$ \\
\hline \multicolumn{2}{|l|}{ Loción para la piel } \\
\hline Etanol $70^{\circ}$ & $40 \%$ \\
\hline Alcohol Isopropílico & $45 \%$ \\
\hline Propilenglicol & $14 \%$ \\
\hline Aceite esencial de naranja & $1 \%$ \\
\hline \multicolumn{2}{|l|}{ Colutorio } \\
\hline Etanol $70^{\circ}$ & $60 \%$ \\
\hline Propilenglicol & $5 \%$ \\
\hline Glicerina & $5 \%$ \\
\hline Ácido cítrico & $2 \%$ \\
\hline Mentol & $0.20 \%$ \\
\hline Aceite esencial de naranja & $1 \%$ \\
\hline Colorante naranja & $0,01 \%$ \\
\hline Agua destilada & $100 \mathrm{~mL}$ \\
\hline
\end{tabular}

\section{RESULTADOS}

\section{Extracción y rendimiento del aceite esencial}

Por el método gravimétrico - volumétrico se determinó el porcentaje de rendimiento del aceite esencial (\%RAE) con la siguiente expresión: 
$\% R A E=$ Vol. $_{A E}(m L) /$ Pmuestra $(g) \times 100$

Donde:

Vol. $_{\text {AE }}$ : Volumen del aceite esencial obtenido; en mililitros

$\mathrm{P}_{\text {muestra }}$ : Peso de la muestra a destilar; en gramos.

El rendimiento de aceite esencial fue de o,4 por ciento v/p.

\section{Análisis preliminar, físicoquímico y composi- ción química}

Los resultados se reportan en las tablas 1 y 2.

Tabla 1. Análisis preliminar y ensayos físicos del aceite esencial

\begin{tabular}{ll}
\hline \multicolumn{1}{c}{ Determinación } & \multicolumn{1}{c}{ Resultados } \\
\hline \multirow{2}{*}{ Análisis organoléptico } & $\begin{array}{l}\text { Líquido oleoso, ligeramente amarillento, } \\
\text { aromático y agradable, volátil y ligera- } \\
\text { mente picante. } \\
\text { Inmiscible en agua }\end{array}$ \\
& $\begin{array}{l}\text { Miscible en alcohol absoluto } \\
\text { niscibilidad }\end{array}$ \\
& $\begin{array}{l}\text { Índice de refracción }\left(20^{\circ} \mathrm{C}\right): 1,112 \\
\text { Gravedad específica }\left(20^{\circ} \mathrm{C}\right): 0,879\end{array}$ \\
& Rotación óptica $\left(20^{\circ} \mathrm{C}\right):-64^{\circ}$ \\
\hline
\end{tabular}

Determinación de la composición química del aceite esencial por CG/EM

Tabla 2. Composición química del aceite esencial (CG/EM)

\begin{tabular}{lc}
\hline \multicolumn{1}{c}{ Componentes químicos } & $\begin{array}{c}\text { Tiempo de retención (TR) } \\
\text { en minutos }\end{array}$ \\
\hline Limoneno & 1,79 \\
$\beta$-Linalol & 2,03 \\
Decanal & 2,61 \\
$2(10)-P i n e n o,(1 S, 5 S)-(-)-$ & 1,55 \\
\hline
\end{tabular}

\section{DISCUSIÓN}

Del pericarpio del fruto de Citrus sinensis L. se obtuvo un rendimiento de aceite esencial de 0,4 por ciento $\mathrm{p} / \mathrm{v}$. Este resultado tiene que ver con las características geográficas y la época y la zona de recolección del fruto, ya que la altitud y el carácter climático tienen efectos en la producción del aceite esencial, considerando que para un material fresco existe un intervalo de 0,07 a 0,20 por ciento de variación de una especie a otra ${ }^{(3,4)}$.

El aceite obtenido por arrastre con vapor de agua, permitió realizar el análisis preliminar y los ensayos fisicoquímicos, entre estos: miscibilidad, densidad, índice de refracción y actividad óptica, que respondieron a las propiedades reportadas para los aceites esenciales y así mismo se puso en evidencia que los componentes químicos del aceite son de baja polaridad, miscibles en solventes orgánicos e inmiscibles en agua ${ }^{(5,6)}$.

La determinación de la composición química, rea-

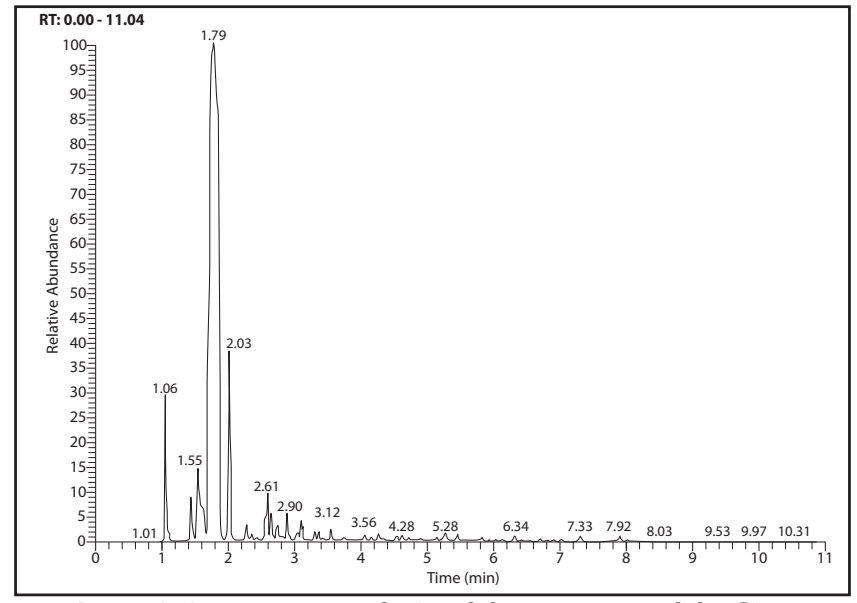

Figura 1. Cromatograma de Gas del aceite esencial de Citrus sinensis $\mathrm{L}$.

lizada por CG/EM, permitió identificar los siguientes componentes químicos: Limoneno, $\beta$-linalol, decanal y 2(10)-pineno, $\left({ }_{1} S,{ }_{5} S\right)-(-)-$. Existen evidencias de que estas sustancias constituyen un grupo de moléculas con actividad biológica ampliamente distribuida en los frutos cítricos y los vegetales, comprendiendo a flavonoides, antraquinonas, cumarinas, cromonas y otros ${ }^{(7,8)}$.

La obtención del aceite esencial de Citrus sinensis L. de o,4 por ciento $\mathrm{p} / \mathrm{v}$ concuerda con otro estudio en el que se obtuvo de $100 \mathrm{~g}$ de cáscaras un rendimiento de 0,35 por ciento $\mathrm{p} / \mathrm{v}{ }^{(9)}$; mientras que en otro estudio, se obtuvo un rendimiento de o, 6 por ciento, dato similar al encontrado en nuestro estudio ${ }^{(10)}$.

También en un estudio químico-bromatológico realizado en semillas de Citrus sinensis L., se verificó la presencia del limoneno y se concluyó que las semillas de este fruto proporcionan un aceite de calidad y sabor agradable, comportándose como un aceite semisecante similar al aceite de las semillas de algodón (11). En el análisis del control micológico en mermeladas de naranja de Citrus sinensis L., se determinó que el recuento de filamentos de hongos y campos positivos expresaba un promedio de 15 por ciento, estando dentro de la tolerancia ${ }^{(12)}$.

Las caries dentales y la enfermedad periodontal están asociadas con los patógenos orales y existen componentes químicos que han sido evaluados contra estos microorganismos. En un estudio realizado por CG/EM en el aceite esencial de Lippia sidoides, arbusto del Brasil, se caracterizó entre otros componentes al timol y carvacrol con propiedades antibacterianas frente a Streptococcus mutans y Candida albicans, ejerciendo prometedores efectos contra los patógenos orales y el crecimiento microbiano oral ${ }^{(13,14)}$. 
La investigación sobre bacterias orales presentes en la placa dental como biofilms y el efecto antibacteriano de los aceites esenciales de Manuka -té del árbol de aceite-, aceite de eucalipto, aceite de lavándula y aceite de rosmarinus en gérmenes orales como: Streptococcus mutans, Streptococcus sobrinus, Fusobacterium nucleatum, Porphyromonas gingivalis y Actinobacillus determinaron que los aceites esenciales inhibieron el crecimiento y la adhesión de Streptococcus mutans en la placa dental ${ }^{(14)}$.

Un estudio realizado sobre la composición química del aceite esencial de hojas de Lantana camara L. reportó predominio de sesquiterpenos, siendo los componentes químicos mayoritarios: (E)-nerolidol (43,4\%), $\gamma$-cadineno $(7,6 \%), \alpha$-humuleno $(4,9 \%)$ y $\beta$-cariofileno $(4,8 \%)$; dando a suponer que la especie analizada representaba un nuevo quimiotipo. Esta especie ejerce efecto de protección sobre Solanum tuberosum (papa), contra Phthorimaea operculella Z. o "polilla de la papa" en almacén ${ }^{(15)}$.

En el estudio realizado sobre el aceite esencial de Croton cajucara Benth se observó actividad leishmanicida, así mismo se demostró que este aceite inhibe el crecimiento de muestras referenciales de Candida albicans, Staphylococcus aureus, Streptoccocus sobrinus, Lactobacillus casei, Porphyromonas gingivalis y Streptoccocus mutans; todos ellos asociados a las enfermedades de la cavidad bucal ${ }^{(16)}$.

\section{CONCLUSIONES}

- En el aceite esencial del pericarpio del Citrus sinensis L. se identificaron los siguientes componente químicos: Limoneno, $\beta$-linalol, decanal y $2(10)$ pineno, $\left({ }_{1} S, 5 S\right)-(-)-$.

- Se determinó que el aceite esencial presenta actividad antibacteriana significativa frente a los microganismos Gram positivos: Staphylococcus aureus ATCC 25933 y Staphylococcus epidermidis cepa clínica, a las concentraciones de 50 y 100 por ciento, no presentando actividad antibacteriana contra Bacillus subtilis cepa clínica, Escherichia coli cepa clínica y Pseudomonas aeruginosa ATCC 27853.

Aceite esencial de Citrus Sinesis L.

Staphylococcus aureus ATCC 25933

Aceite esencial de Citrus Sinesis L. Staphylococcus epidermidis (cepa clínica)

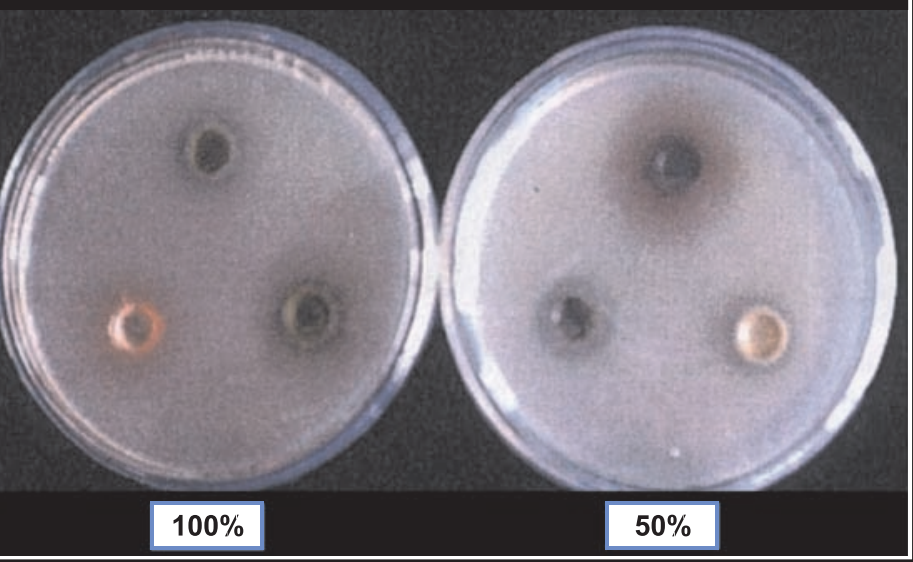

Figura 2. Actividad antibacteriana del aceite esencial de Citrus sinensis L.

- Para el caso de las formulaciones gel, loción y colutorio, además de las pruebas de estabilidad acelerada, según lo establecido por la USP 32/NF27 y la Sociedad Española de Farmacia Hospitalaria ${ }^{(17,18)}$, también se sometieron a pruebas de tolerancia e irritabilidad. Finalmente, las formulaciones fueron sometidas a pruebas, tanto en voluntarios sanos o con alguna patología, en piel y cavidad bucal; demostrándose que en ambos casos toleran las formulaciones sin producirse ningún tipo de efecto no deseado.

\section{REFERENCIAS BIBLIOGRÁFICAS}

1. Rojas R, Bustamante B, Bauer J, Fernández I, Albán J, Lock O. Antimicrobial activity of selected peruvian medicinal plants. J Ethnopharmacol 2003; 88(2-3): 199-204.

2. Magaldi S, Matta-Essayag S, Hartung de Capriles C, Perez C, Colella MT, Olaizola C, et al. Difusion for antifungal susceptibility testing. Int J Infect Dis 2004; 8(1):39-45.

3. AD Asociación y Desarrollo. Hierbas aromáticas y medicinales en el distrito de San Miguel de El Faique. Huancabamba-Misión rural. Piura 1996; 2:6-7.

4. Biondi D, Cianci P, Geraci C, Ruberto G, Piatelli M. Antimicrobial activity and chemical composition of essential oils. Flavor and Fragance Journal 1993; 8(6): 331-7.

5. Lock de Ugaz O. Investigación Fitoquímica. Editorial de 
la Pontificia Universidad Católica del Perú. Lima, 1994.

6. Dominguez X. Método de Investigación Fitoquímica. za Ed. 1985. Editorial Limusa. México DF.

7. Van Acquire SA, Van den Berg DJ, Tromp MN, Griffioen $\mathrm{DH}$, Van Bennekom WP, Vander Vight WJ, et al. Estructural Aspects of Antioxidan activity of flavonoids. Free Radic Biol Med 1996; 20:331-42.

8. De Groot H, Raven U. Tissue injury by reactive oxygen species and the protective effects of flavonoids. Fundam Clin Pharmacol 1998; 12(3): 249-55.

9. Chávez AA. Extracción industrial de esencia de cáscaras de naranja y preparación de derivados del limoneno. Tesis para optar el grado de bachiller en Farmacia y Bioquímica. UNMSM. Lima, 1952.

10. Flores FT. Investigación de esencia de vainilla, anís, naranja y su identificación mediante cromatografía y espectrofotometría infrarroja. Tesis para optar al Título Profesional de Químico Farmacéutico. UNMSM. Lima, 1995.

11. KikushimaYE. Estudio Químico-bromatológicodelas semillas de Citrus sinensis L. Tesis para optaral Título Profesional de Químico Farmacéutico. UNMSM. Lima, 1984.

12. Malamud WF. Control micológico en mermeladas de fresa (Fragaria vesca) y naranja (Citrus aurantium y Citrus sinensis L.) Tesis para optar el grado de bachiller en Farmacia y Bioquímica. UNMSM. Lima, 1967.

13. Botelho MA et al. Antimicrobial activity essential oil from Lippia sidoides, carvacrol and thymol against oral pathogens. J Med Biol Res 2007; 40(3): 349-56.
14. Takarada K, Kimizuka R, Takahashi N, Honma K, Okuda K, Kato T. A comparison of the antibacterial efficacies of essential oils against oral pathogens. Oral Microbiol Immunol 2004; 19(1): 61-4.

15. Laksman L. Potato tuber moth, Phthorimaea operculella (Zeller) in North Eastern Hills region and a simple method for is control. Indian Journal of Agricultural Sciences. 1988; 58(2): 130-132.

16. Alviano WS, Mendoca-Filho RR, Aviano DS, Bizzo HR, Souto-Padrón T, et al. Actividad Antimicrobiana de Croton Cajucara Benth linalol. Oral Microbiol Immunol. 2005; 20(2): 101-5.

17. Farmacopea de los Estados Unidos de América y Formulario Nacional (USP 32 - NF 27). The United States Pharmacopeial Convention. Maryland 2009.

Manuscrito recibido el: 05/02/2010

Aceptado para su publicación el: 08/04/2010

\section{Correspondencia:}

Nombre: Dr. José Juárez Eyzaguirre

Dirección: Jr. Los Tulipanes 343 - Lima 12 / Jr. Puno 1002 - Lima o1 - Perú

e-mail: jjuareze@unmsm.edu.pe 